\title{
Implementing Likelihood-Based Inference for Fat-Tailed Distributions
}

\author{
M. Rekkas* A. Wong ${ }^{\dagger}$
}

\begin{abstract}
The theoretical advancements in higher order likelihood-based inference methods have been tremendous over the past two decades. The application of these methods in the applied literature however has been far from widespread. A critical barrier to adoption has likely been the computational difficulties associated with the implementation of these methods. This paper provides the applied researcher with a systematic exposition of the calculations and computer code required to implement the higher order conditional inference methodology of Fraser and Reid (1995) for problems involving heavy- or fat-tailed distributions.
\end{abstract}

Key words: third-order inference; p-values; likelihood; fat-tailed distributions

${ }^{*}$ Corresponding author. Department of Economics, Simon Fraser University, 8888 University Drive, Burnaby, British Columbia V5A 1S6, email: mrekkas@sfu.ca, phone: (778) 782-6793, fax: (778) 782-5944

${ }^{\dagger}$ Department of Mathematics and Statistics, York University, 4700 Keele Street, Toronto, Ontario M3J 1P3

We would like to thank an anonymous referee and the editor for helpful suggestions. We gratefully acknowledge the support of the Natural Sciences and Engineering Research Council of Canada. 


\section{Introduction}

The analysis of financial data often requires the specification of a suitably defined heavy-tailed distribution. While standard first-order asymptotic-based tests are available, their use on such non-normal distributions may produce results which are severely inaccurate, with this inaccuracy exacerbated when the data is limited. In terms of higher order tests, the theoretical literature has seen tremendous advances in higher order likelihood-based methods over the past two decades. The adoption of these tests in the applied literature however, has seriously lagged behind. The main barrier to adoption is likely the (perceived) difficulty of implementing these higher order methodologies.

In this paper our goal is to address this impediment. We consider the higher order likelihood-based inference methodology proposed in Fraser and Reid (1995) and show the applied researcher how to use it in practice for three commonly encountered fat-tailed distributions, the $t$, the Cauchy, and the Pareto distributions. We provide a much needed systematic step-by-step exposition of the calculations required to undertake the higher order methodology and make computer code written in $\mathrm{R}$ available. It is noted that Brazzale (1999) bridges the gap between theory and application by providing SPlus code for approximate conditional inference for logistic and loglinear models. In what follows below we present an overview of the methodology and provide the requisite components and computer code necessary for implementation. In particular, Section 2 provides a brief description of first- and third-order likelihood methods and Section 3 details the necessary required components for the $t$ distribution. Sections 4 and 5 provide the components for the Cauchy and Pareto distributions, respectively. Section 6 concludes.

\section{Higher Order Asymptotics}

For a sample $y=\left(y_{1}, y_{2}, \ldots, y_{n}\right)^{\prime}$, with $\theta=\left(\psi, \lambda^{\prime}\right)^{\prime}$, where $\psi$ is the one-dimensional component parameter of interest and $\lambda$ is the $p-1$ dimensional nuisance parameter, let the log-likelihood function be denoted by $l(\theta ; y)$ or more simply as $l(\theta)$. The two most familiar likelihood-based methods that are used for testing the scalar parameter $\psi=\psi(\theta)=\psi_{0}$ are the Wald departure and the signed log-likelihood departure:

$$
\begin{aligned}
q & =\left(\hat{\psi}-\psi_{0}\right)\left\{j^{\psi \psi}(\hat{\theta})\right\}^{-1 / 2} \\
r & =\operatorname{sgn}\left(\hat{\psi}-\psi_{0}\right)\left[2\left\{l(\hat{\theta})-l\left(\hat{\theta}_{\psi_{0}}\right)\right\}\right]^{1 / 2},
\end{aligned}
$$

where $\hat{\theta}=\left(\hat{\psi}, \hat{\lambda}^{\prime}\right)^{\prime}$ is the maximum likelihood estimate obtained by solving the first-order conditions

$$
l_{\theta}(\hat{\theta})=l_{\theta}(\hat{\theta} ; y)=\left.\frac{\partial l(\theta)}{\partial \theta}\right|_{\hat{\theta}}=0
$$


and $\hat{\theta}_{\psi}=\left(\psi, \hat{\lambda}_{\psi}^{\prime}\right)^{\prime}$ is the constrained maximum likelihood estimate obtained by maximizing $l(\theta)$ over $\lambda$ while treating $\psi$ fixed. The information matrix is given by

$$
j_{\theta \theta^{\prime}}(\theta)=\left[\begin{array}{cc}
-\frac{\partial^{2} l(\theta)}{\partial \psi \partial \psi} & -\frac{\partial^{2} l(\theta)}{\partial \psi \partial \lambda^{\prime}} \\
-\frac{\partial^{2} l(\theta)}{\partial \psi \partial \lambda^{\prime}} & -\frac{\partial^{2} l(\theta)}{\partial \lambda \partial \lambda^{\prime}}
\end{array}\right]=\left[\begin{array}{cc}
-l_{\psi \psi}(\theta) & -l_{\psi \lambda^{\prime}}(\theta) \\
-l_{\psi \lambda^{\prime}}(\theta) & -l_{\lambda \lambda^{\prime}}(\theta)
\end{array}\right]=\left[\begin{array}{cc}
j_{\psi \psi}(\theta) & j_{\psi \lambda^{\prime}}(\theta) \\
j_{\psi \lambda^{\prime}}(\theta) & j_{\lambda \lambda^{\prime}}(\theta)
\end{array}\right] .
$$

The observed information matrix evaluated at $\hat{\theta}$ is denoted as $j_{\theta \theta^{\prime}}(\hat{\theta})$. An estimated asymptotic variance of $\hat{\theta}$ is then given by

$$
j^{\theta \theta^{\prime}}(\hat{\theta})=\left\{j_{\theta \theta^{\prime}}(\hat{\theta})\right\}^{-1}=\left[\begin{array}{ll}
j^{\psi \psi}(\hat{\theta}) & j^{\psi \lambda^{\prime}}(\hat{\theta}) \\
j^{\psi \lambda^{\prime}}(\hat{\theta}) & j^{\lambda \lambda^{\prime}}(\hat{\theta})
\end{array}\right] .
$$

P-values using $q$ and $r$ can be approximated by $\Phi(q)$ and $\Phi(r)$, where $\Phi(\cdot)$ is the standard normal distribution function. These methods have order of convergence $O\left(n^{-1 / 2}\right)$ and are referred to as first-order methods.

Based on Barndorff-Nielsen (1986, 1991), Fraser and Reid (1995) have developed a methodology that achieves improvements to the signed log-likelihood departure. Fraser and Reid show that this method achieves a known $O\left(n^{-3 / 2}\right)$ rate of convergence and as such is referred to as a third-order method. The general theory is based on conditional evaluations and involves techniques closely related to the saddlepoint approach. The method involves a dimension reduction from the dimension of the variable $y$ to the dimension of the parameter $\theta$. This does not need to be determined explicitly, tangent vectors to the conditioning variable at the data itself are sufficient. The tangent ancillary vectors are obtained at the observed data through a full-dimensional pivotal quantity $z(\theta ; y)$ :

$$
V=\left.\frac{\partial y}{\partial \theta^{\prime}}\right|_{\hat{\theta}}=\left.\left\{\frac{\partial z(y, \theta)}{\partial y^{\prime}}\right\}^{-1}\left\{\frac{\partial z(y, \theta)}{\partial \theta^{\prime}}\right\}\right|_{\hat{\theta}} .
$$

The essential component of the methodology is the locally defined canonical parameter at the observed data:

$$
\varphi^{\prime}(\theta)=\frac{\partial}{\partial V} l(\theta ; y)=\frac{\partial}{\partial y} l(\theta ; y) \cdot V .
$$

This new parameter $\varphi(\theta)$ represents a very special parameterization; $\varphi(\theta)$ is the canonical parameter of an approximating exponential model. The nuisance parameters of the model can then be eliminated using a marginalization procedure. This involves the parameter of interest $\psi(\theta)$ being re-scaled on the $\varphi(\theta)$ dimension

$$
\chi(\theta)=\frac{\psi_{\varphi^{\prime}}\left(\hat{\theta}_{\psi}\right)}{\left|\psi_{\varphi^{\prime}}\left(\hat{\theta}_{\psi}\right)\right|} \varphi(\theta)
$$

where $\psi_{\varphi^{\prime}}(\theta)=\partial \psi(\theta) / \partial \varphi^{\prime}=\left(\partial \psi(\theta) / \partial \theta^{\prime}\right)\left(\partial \varphi(\theta) / \partial \theta^{\prime}\right)^{-1}=\psi_{\theta^{\prime}}(\theta) \varphi_{\theta^{\prime}}^{-1}(\theta)$. This reparameterization is used to obtain the departure measure $Q$ :

$$
Q=\operatorname{sgn}(\hat{\psi}-\psi)\left|\chi(\hat{\theta})-\chi\left(\hat{\theta}_{\psi}\right)\right|\left\{\frac{\left|\hat{\jmath}_{\varphi \varphi^{\prime}}(\hat{\theta})\right|}{\left|\hat{\jmath}_{\left(\lambda \lambda^{\prime}\right)}\left(\hat{\theta}_{\psi}\right)\right|}\right\}^{1 / 2},
$$


where $\hat{\jmath}_{\varphi \varphi^{\prime}}$ and $\hat{\jmath}_{\left(\lambda \lambda^{\prime}\right)}$ are the observed information matrix evaluated at $\hat{\theta}$ and observed nuisance information matrix evaluated at $\hat{\theta}_{\psi}$, respectively, calculated in the locally defined canonical parameter, $\varphi(\theta)$, scale. Fraser and Reid (1995) show that their determinants can be obtained as follows:

$$
\begin{aligned}
\left|\hat{\jmath}_{\varphi \varphi^{\prime}}(\hat{\theta})\right| & =\left|\hat{\jmath}_{\theta \theta^{\prime}}(\hat{\theta})\right|\left|\varphi_{\theta^{\prime}}(\hat{\theta})\right|^{-2} \\
\left|\hat{\jmath}_{\left(\lambda \lambda^{\prime}\right)}\left(\hat{\theta}_{\psi}\right)\right| & =\left|\hat{\jmath}_{\lambda \lambda^{\prime}}\left(\hat{\theta}_{\psi}\right)\right|\left|\varphi_{\lambda}^{\prime}\left(\hat{\theta}_{\psi}\right) \varphi_{\lambda}\left(\hat{\theta}_{\psi}\right)\right|^{-1} .
\end{aligned}
$$

Third-order accurate p-value approximations can be obtained by combining the signed log-likelihood ratio given in (2) and the particular maximum likelihood departure from (9) using either:

$$
\Phi(r)+\phi(r)\left\{\frac{1}{r}-\frac{1}{Q}\right\}
$$

or

$$
\Phi\left(r^{*}\right)=\Phi\left(r-r^{-1} \log \frac{r}{Q}\right),
$$

due respectively to Lugannani and Rice (1980) and Barndorff-Nielsen (1991); $\phi$ is the standard normal density. These approximations are asymptotically equivalent and have $O\left(n^{-3 / 2}\right)$ accuracy.

When faced with implementing this methodology to produce these highly accurate p-values, the applied statistician may find herself weighing the benefits of increased accuracy against the costs of learning how to transform the theory into practice. In the next three sections, we consider using this method in practice for the $t$ distribution, the Cauchy distribution, and the Pareto distribution.

\section{The $t$ Distribution}

The superiority of the third-order method for the $t$ distribution has been shown through simulations in Taback (2002), Fraser et al. (1999), and Bedard et al. (2007). The mechanics of using the method for the applied researcher however have not been available. We provide the essential ingredients required to implement the higher order methodology. Consider the simple regression model

$$
y=\alpha+\beta x+\sigma \epsilon
$$

where $\epsilon$ is distributed as Student's $t$ with 7 degrees of freedom or $\epsilon \sim t_{7}$. Suppose the researcher is interested in inference on $\beta$, so that $\psi(\theta)=\beta$. In order to obtain p-values using the Lugannani and Rice or BarndorffNielsen expressions in (12) and (13), a few key results must be available.

We start with the probability density function for $\epsilon$, this function is given by $f(\epsilon)$,

$$
\begin{aligned}
f(\epsilon) & =\frac{\Gamma(4)}{\Gamma(1 / 2) \Gamma(7 / 2)}\left(1+\frac{\epsilon^{2}}{7}\right)^{-4} \frac{1}{\sqrt{7}} \\
& =\frac{\Gamma(4)}{\Gamma(1 / 2) \Gamma(7 / 2)} \frac{7^{4}}{\sqrt{7}} \frac{1}{\left(7+\epsilon^{2}\right)^{4}},
\end{aligned}
$$


where $\Gamma(\cdot)$ represents the Gamma function. Given this density function, the probability function for $y$ is derived as $f(y ; \alpha, \beta, \sigma)$,

$$
\begin{aligned}
f(y ; \alpha, \beta, \sigma) & =\frac{\Gamma(4)}{\Gamma(1 / 2) \Gamma(7 / 2)} \frac{7^{4}}{\sqrt{7}}\left[7+\left(\frac{y-\alpha-\beta x}{\sigma}\right)^{2}\right]^{-4} \frac{1}{\sigma} \\
& =\frac{\Gamma(4)}{\Gamma(1 / 2) \Gamma(7 / 2)} \frac{7^{4}}{\sqrt{7}}\left[7 \sigma^{2}+(y-\alpha-\beta x)^{2}\right]^{-4} \sigma^{7} .
\end{aligned}
$$

Given a sample of data, $y=\left(y_{1}, y_{2}, \ldots, y_{n}\right)^{\prime}$, the likelihood function can be specified as

$$
L(\alpha, \beta, \sigma)=c \sigma^{7 n} \prod_{i=1}^{n}\left[7 \sigma^{2}+\left(y_{i}-\alpha-\beta x_{i}\right)^{2}\right]^{-4}
$$

and the log-likelihood function is denoted by $l(\alpha, \beta, \sigma)$,

$$
l(\alpha, \beta, \sigma)=7 n \log \sigma-4 \sum_{i=1}^{n} \log \left[7 \sigma^{2}+\left(y_{i}-\alpha-\beta x_{i}\right)^{2}\right] .
$$

The maximum likelihood estimate $\hat{\theta}=(\hat{\alpha}, \hat{\beta}, \hat{\sigma})^{\prime}$ is obtained by solving the normal equations from the first derivatives:

$$
\begin{aligned}
& l_{\alpha}=8 \sum_{i=1}^{n} \frac{y_{i}-\alpha-\beta x_{i}}{7 \sigma^{2}+\left(y_{i}-\alpha-\beta x_{i}\right)^{2}} \\
& l_{\beta}=8 \sum_{i=1}^{n} \frac{\left(y_{i}-\alpha-\beta x_{i}\right) x_{i}}{7 \sigma^{2}+\left(y_{i}-\alpha-\beta x_{i}\right)^{2}} \\
& l_{\sigma}=\frac{7 n}{\sigma}-56 \sum_{i=1}^{n} \frac{\sigma}{7 \sigma^{2}+\left(y_{i}-\alpha-\beta x_{i}\right)^{2}} .
\end{aligned}
$$

The second derivatives of the log-likelihood are needed to construct the information matrix denoted by $j_{\theta \theta^{\prime}}(\theta)$ :

$$
\begin{aligned}
& l_{\alpha \alpha}=8 \sum_{i=1}^{n} \frac{-7 \sigma^{2}+\left(y_{i}-\alpha-\beta x_{i}\right)^{2}}{\left[7 \sigma^{2}+\left(y_{i}-\alpha-\beta x_{i}\right)^{2}\right]^{2}} \\
& l_{\alpha \beta}=8 \sum_{i=1}^{n} \frac{-7 \sigma^{2} x_{i}+\left(y_{i}-\alpha-\beta x_{i}\right)^{2} x_{i}}{\left[7 \sigma^{2}+\left(y_{i}-\alpha-\beta x_{i}\right)^{2}\right]^{2}} \\
& l_{\alpha \sigma}=8 \sum_{i=1}^{n} \frac{-14\left(y_{i}-\alpha-\beta x_{i}\right) \sigma}{\left[7 \sigma^{2}+\left(y_{i}-\alpha-\beta x_{i}\right)^{2}\right]^{2}} \\
& l_{\beta \beta}=8 \sum_{i=1}^{n} \frac{-7 \sigma^{2} x_{i}^{2}+\left(y_{i}-\alpha-\beta x_{i}\right)^{2} x_{i}^{2}}{\left[7 \sigma^{2}+\left(y_{i}-\alpha-\beta x_{i}\right)^{2}\right]^{2}} \\
& l_{\beta \sigma}=8 \sum_{i=1}^{n} \frac{-14\left(y_{i}-\alpha-\beta x_{i}\right) x_{i} \sigma}{\left[7 \sigma^{2}+\left(y_{i}-\alpha-\beta x_{i}\right)^{2}\right]^{2}} \\
& l_{\sigma \sigma}=-\frac{7 n}{\sigma^{2}}-56 \sum_{i=1}^{n} \frac{-7 \sigma^{2}+\left(y_{i}-\alpha-\beta x_{i}\right)^{2}}{\left[7 \sigma^{2}+\left(y_{i}-\alpha-\beta x_{i}\right)^{2}\right]^{2}} .
\end{aligned}
$$

The information matrix evaluated at the maximum likelihood estimate produces the observed information 
matrix denoted by $j_{\theta \theta^{\prime}}(\hat{\theta})$.

For the canonical parameterization, $\varphi(\theta)$, tangent ancillary directions and sample space derivatives are required and obtained as follows:

$$
\begin{aligned}
& V=\left.\frac{\partial y}{\partial \theta^{\prime}}\right|_{\hat{\theta}}=\left.\left(\frac{\partial y}{\partial \alpha}, \frac{\partial y}{\partial \beta}, \frac{\partial y}{\partial \sigma}\right)\right|_{\hat{\theta}}=\left.(1, x, \epsilon)\right|_{\hat{\theta}} \\
& =\left(\begin{array}{lll}
1 & x & \frac{y-\hat{\alpha}-\hat{\beta} x}{\hat{\sigma}}
\end{array}\right) \\
& \frac{\partial l}{\partial y_{i}}=-8\left[\frac{y_{i}-\alpha-\beta x_{i}}{7 \sigma^{2}+\left(y_{i}-\alpha-\beta x_{i}\right)^{2}}\right] .
\end{aligned}
$$

The reparameterization is given by

$$
\begin{aligned}
\varphi^{\prime}(\theta) & =\left[\frac{\partial l}{\partial y}\right]\left[\begin{array}{lll}
1 & x & \frac{y-\hat{\alpha}-\hat{\beta} x}{\hat{\sigma}}
\end{array}\right] \\
& =\left[-8 \sum_{i=1}^{n} \frac{y_{i}-\alpha-\beta x_{i}}{7 \sigma^{2}+\left(y_{i}-\alpha-\beta x_{i}\right)^{2}},-8 \sum_{i=1}^{n} \frac{\left(y_{i}-\alpha-\beta x_{i}\right) x_{i}}{7 \sigma^{2}+\left(y_{i}-\alpha-\beta x_{i}\right)^{2}},\right. \\
& \left.=-8 \sum_{i=1}^{n} \frac{\left(y_{i}-\alpha-\beta x_{i}\right)}{7 \sigma^{2}+\left(y_{i}-\alpha-\beta x_{i}\right)^{2}} \cdot \frac{y_{i}-\hat{\alpha}-\hat{\beta} x_{i}}{\hat{\sigma}}\right],
\end{aligned}
$$

with elements denoted as

$$
\begin{aligned}
\varphi_{1}(\theta) & =-8 \sum_{i=1}^{n} \frac{y_{i}-\alpha-\beta x_{i}}{7 \sigma^{2}+\left(y_{i}-\alpha-\beta x_{i}\right)^{2}} \\
\varphi_{2}(\theta) & =-8 \sum_{i=1}^{n} \frac{\left(y_{i}-\alpha-\beta x_{i}\right) x_{i}}{7 \sigma^{2}+\left(y_{i}-\alpha-\beta x_{i}\right)^{2}} \\
\varphi_{3}(\theta) & =-8 \sum_{i=1}^{n} \frac{y_{i}-\alpha-\beta x_{i}}{7 \sigma^{2}+\left(y_{i}-\alpha-\beta x_{i}\right)^{2}} \cdot \frac{y_{i}-\hat{\alpha}-\hat{\beta} x_{i}}{\hat{\sigma}} .
\end{aligned}
$$

Using these elements, the matrix $\varphi_{\theta}(\theta)$ is computed

$$
\varphi_{\theta}(\theta)=\left[\begin{array}{ccc}
\varphi_{1 \alpha}(\theta) & \varphi_{1 \beta}(\theta) & \varphi_{1 \sigma}(\theta) \\
\varphi_{2 \alpha}(\theta) & \varphi_{2 \beta}(\theta) & \varphi_{2 \sigma}(\theta) \\
\varphi_{3 \alpha}(\theta) & \varphi_{3 \beta}(\theta) & \varphi_{3 \sigma}(\theta)
\end{array}\right],
$$

where

$$
\begin{aligned}
& \varphi_{1 \alpha}(\theta)=\frac{\partial \varphi_{1}(\theta)}{\partial \alpha}=-8 \sum_{i=1}^{n} \frac{-7 \sigma^{2}+\left(y_{i}-\alpha-\beta x_{i}\right)^{2}}{\left[7 \sigma^{2}+\left(y_{i}-\alpha-\beta x_{i}\right)^{2}\right]^{2}} \\
& \varphi_{1 \beta}(\theta)=\frac{\partial \varphi_{1}(\theta)}{\partial \beta}=-8 \sum_{i=1}^{n} \frac{-7 \sigma^{2} x_{i}+\left(y_{i}-\alpha-\beta x_{i}\right)^{2} x_{i}}{\left[7 \sigma^{2}+\left(y_{i}-\alpha-\beta x_{i}\right)^{2}\right]^{2}} \\
& \varphi_{1 \sigma}(\theta)=\frac{\partial \varphi_{1}(\theta)}{\partial \sigma}=-8 \sum_{i=1}^{n} \frac{-14\left(y_{i}-\alpha-\beta x_{i}\right) \sigma}{\left[7 \sigma^{2}+\left(y_{i}-\alpha-\beta x_{i}\right)^{2}\right]^{2}} \\
& \varphi_{2 \alpha}(\theta)=\frac{\partial \varphi_{2}(\theta)}{\partial \alpha}=-8 \sum_{i=1}^{n} \frac{-7 \sigma^{2} x_{i}+\left(y_{i}-\alpha-\beta x_{i}\right)^{2} x_{i}}{\left[7 \sigma^{2}+\left(y_{i}-\alpha-\beta x_{i}\right)^{2}\right]^{2}}
\end{aligned}
$$




$$
\begin{aligned}
& \varphi_{2 \beta}(\theta)=\frac{\partial \varphi_{2}(\theta)}{\partial \beta}=-8 \sum_{i=1}^{n} \frac{-7 \sigma^{2} x_{i}^{2}+\left(y_{i}-\alpha-\beta x_{i}\right)^{2} x_{i}^{2}}{\left[7 \sigma^{2}+\left(y_{i}-\alpha-\beta x_{i}\right)^{2}\right]^{2}} \\
& \varphi_{2 \sigma}(\theta)=\frac{\partial \varphi_{2}(\theta)}{\partial \sigma}=-8 \sum_{i=1}^{n} \frac{-14\left(y_{i}-\alpha-\beta x_{i}\right) x_{i} \sigma}{\left[7 \sigma^{2}+\left(y_{i}-\alpha-\beta x_{i}\right)^{2}\right]^{2}} \\
& \varphi_{3 \alpha}(\theta)=\frac{\partial \varphi_{3}(\theta)}{\partial \alpha}=-8 \sum_{i=1}^{n} \frac{-7 \sigma^{2}+\left(y_{i}-\alpha-\beta x_{i}\right)^{2}}{\left[7 \sigma^{2}+\left(y_{i}-\alpha-\beta x_{i}\right)^{2}\right]^{2}} \cdot \frac{y_{i}-\hat{\alpha}-\hat{\beta} x_{i}}{\hat{\sigma}} \\
& \varphi_{3 \alpha}(\theta)=\frac{\partial \varphi_{3}(\theta)}{\partial \beta}=-8 \sum_{i=1}^{n} \frac{-7 \sigma^{2} x_{i}+\left(y_{i}-\alpha-\beta x_{i}\right)^{2} x_{i}}{\left[7 \sigma^{2}+\left(y_{i}-\alpha-\beta x_{i}\right)^{2}\right]^{2}} \cdot \frac{y_{i}-\hat{\alpha}-\hat{\beta} x_{i}}{\hat{\sigma}} \\
& \varphi_{1 \beta}(\theta)=\frac{\partial \varphi_{3}(\theta)}{\partial \sigma}=-8 \sum_{i=1}^{n} \frac{-14\left(y_{i}-\alpha-\beta x_{i}\right) \sigma}{\left[7 \sigma^{2}+\left(y_{i}-\alpha-\beta x_{i}\right)^{2}\right]^{2}} \cdot \frac{y_{i}-\hat{\alpha}-\hat{\beta} x_{i}}{\hat{\sigma}} .
\end{aligned}
$$

The stand-in scalar canonical parameter can then be obtained as

$$
\chi(\theta)=\frac{\psi_{\varphi^{\prime}}\left(\hat{\theta}_{\psi}\right)}{\left\|\psi_{\varphi^{\prime}}\left(\hat{\theta}_{\psi}\right)\right\|} \varphi(\theta),
$$

where

$$
\psi_{\varphi^{\prime}}(\theta)=\psi_{\theta^{\prime}}(\theta) \varphi_{\theta^{\prime}}^{-1}(\theta)
$$

and

$$
\psi_{\theta}(\theta)=\left(\begin{array}{lll}
0 & 1 & 0
\end{array}\right) .
$$

Using these ingredients above, one can now compute the Wald-type departure measure given by $Q$ in (9),

$$
Q=\operatorname{sgn}(\hat{\psi}-\psi)\left|\chi(\hat{\theta})-\chi\left(\hat{\theta}_{\psi}\right)\right|\left\{\frac{\left|\hat{\jmath}_{\varphi \varphi^{\prime}}(\hat{\theta})\right|}{\left|\hat{\jmath}_{\left(\lambda \lambda^{\prime}\right)}\left(\hat{\theta}_{\psi}\right)\right|}\right\}^{1 / 2},
$$

where recall from (10) and (11), the recalibrated determinants are defined as

$$
\begin{aligned}
\left|\hat{\jmath}_{\varphi \varphi^{\prime}}(\hat{\theta})\right| & =\left|\hat{\jmath}_{\theta \theta^{\prime}}(\hat{\theta})\right|\left|\varphi_{\theta^{\prime}}(\hat{\theta})\right|^{-2} \\
\left|\hat{\jmath}_{\left(\lambda \lambda^{\prime}\right)}\left(\hat{\theta}_{\psi}\right)\right| & =\left|\hat{\jmath}_{\lambda \lambda^{\prime}}\left(\hat{\theta}_{\psi}\right)\right|\left|\varphi_{\lambda}^{\prime}\left(\hat{\theta}_{\psi}\right) \varphi_{\lambda}\left(\hat{\theta}_{\psi}\right)\right|^{-1} .
\end{aligned}
$$

Using the $Q$ in (9) and the $r$ in (2) in the expressions given in (12) and (13) produce p-values whose distributions have third-order accuracy. The exposition for the Cauchy and Pareto distributions are provided in the following two sections in analogous fashion.

\section{The Cauchy Distribution}

The accuracy of the third-order methodology applied to the Cauchy distribution has been considered most recently in Brazzale et al. (2007). Brazzale et al. consider a simple example for a single observation from a Cauchy distribution. As the exact distribution for this case is known, the accuracy of the third-order p-value approximations is easily assessed. These higher order p-value approximations were found to be extremely 
accurate while the p-value produced from a first-order method performed very poorly. In this section, we consider the linear regression model with Cauchy errors as well as two examples.

Consider the regression model

$$
y=\alpha+\beta x+\sigma \epsilon,
$$

where $\epsilon$ is distributed as Cauchy with probability density function

$$
f(\epsilon)=\frac{1}{\pi\left(1+\epsilon^{2}\right)} .
$$

The probability density for $y$ is then derived as

$$
f(y ; \alpha, \beta, \sigma)=\frac{1}{\pi\left[1+\left(\frac{y-\alpha-\beta x}{\sigma}\right)^{2}\right]} \frac{1}{\sigma} .
$$

With data given by $y=\left(y_{1}, y_{2}, \ldots, y_{n}\right)^{\prime}$, the likelihood function is given as

$$
L(\alpha, \beta, \sigma)=\sigma^{-n}-\pi^{-n} \prod_{i=1}^{n}\left[1+\left(\frac{y_{i}-\alpha-\beta x_{i}}{\sigma}\right)^{2}\right]^{-1}
$$

and the log-likelihood function is denoted by $l(\alpha, \beta, \sigma)$,

$$
l(\alpha, \beta, \sigma)=-n \log \sigma-n \log \pi-\sum_{i=1}^{n} \log \left[1+\left(\frac{y_{i}-\alpha-\beta x_{i}}{\sigma}\right)^{2}\right] .
$$

For ease of exposition, we define

$$
z_{i}=\frac{y_{i}-\alpha-\beta x_{i}}{\sigma}
$$

which then leads to

$$
l(\alpha, \beta, \sigma)=-n \log \sigma-n \log \pi-\sum_{i=1}^{n} \log \left(1+z_{i}^{2}\right) .
$$

The maximum likelihood estimate $\hat{\theta}=(\hat{\alpha}, \hat{\beta}, \hat{\sigma})^{\prime}$ is obtained by solving the normal equations from the first derivatives:

$$
\begin{aligned}
& l_{\alpha}=2 \sigma^{-1} \sum_{i=1}^{n}\left(1+z_{1}^{2}\right)^{-1} z_{i} \\
& l_{\beta}=2 \sigma^{-1} \sum_{i=1}^{n}\left(1+z_{1}^{2}\right)^{-1} z_{i} x_{i} \\
& l_{\sigma}=-n \sigma^{-1}+2 \sigma^{-1} \sum_{i=1}^{n}\left(1+z_{1}^{2}\right)^{-1} z_{i}^{2} .
\end{aligned}
$$

The following second derivatives evaluated at the maximum likelihood estimate are used to construct the 
observed information matrix denoted by $j_{\theta \theta^{\prime}}(\hat{\theta})$ :

$$
\begin{aligned}
& l_{\alpha \alpha}=2 \sigma^{-2} \sum_{i=1}^{n}\left[2\left(1+z_{i}^{2}\right)^{-2} z_{i}^{2}-\left(1+z_{i}^{2}\right)^{-1}\right] \\
& l_{\alpha \beta}=2 \sigma^{-2} \sum_{i=1}^{n}\left[2\left(1+z_{i}^{2}\right)^{-2} z_{i}^{2} x_{i}-\left(1+z_{i}^{2}\right)^{-1} x_{i}\right] \\
& l_{\alpha \sigma}=4 \sigma^{-2} \sum_{i=1}^{n}\left[\left(1+z_{i}^{2}\right)^{-2} z_{i}^{3}-\left(1+z_{i}^{2}\right)^{-1} z_{i}\right] \\
& l_{\beta \beta}=2 \sigma^{-2} \sum_{i=1}^{n}\left[2\left(1+z_{i}^{2}\right)^{-2} z_{i}^{2} x_{i}^{2}-\left(1+z_{i}^{2}\right)^{-1} x_{i}^{2}\right] \\
& l_{\beta \sigma}=4 \sigma^{-2} \sum_{i=1}^{n}\left[\left(1+z_{i}^{2}\right)^{-2} z_{i}^{3} x_{i}-\left(1+z_{i}^{2}\right)^{-1} z_{i} x_{i}\right] \\
& l_{\sigma \sigma}=n \sigma^{-2}-2 \sigma^{-2} \sum_{i=1}^{2}\left(1+z_{i}^{2}\right) z_{i}^{2}+4 \sigma^{-2} \sum_{i=1}^{n}\left[\left(1+z_{i}^{2}\right)^{-2} z_{i}^{4}-\left(1+z_{i}^{2}\right)^{-2} z_{i}^{2}\right] .
\end{aligned}
$$

For the canonical parameterization, tangent ancillary directions and sample space derivatives are required and obtained as follows:

$$
\begin{aligned}
V & =\left.\frac{\partial y}{\partial \theta^{\prime}}\right|_{\hat{\theta}}=\left.\left(\frac{\partial y}{\partial \alpha}, \frac{\partial y}{\partial \beta}, \frac{\partial y}{\partial \sigma}\right)\right|_{\hat{\theta}}=\left.(1, x, \epsilon)\right|_{\hat{\theta}}=\left(\begin{array}{lll}
1 & x & \hat{z}
\end{array}\right) \\
& =\frac{\partial l}{\partial y_{i}}=-2 \sigma^{-1}\left(1+z_{i}^{2}\right)^{-1} z_{i} .
\end{aligned}
$$

The reparameterization is given by

$$
\begin{aligned}
\varphi^{\prime}(\theta) & =\left[\frac{\partial l}{\partial y}\right]\left[\begin{array}{lll}
1 & x & \hat{z}
\end{array}\right] \\
& =\left[-2 \sigma^{-1} \sum_{i=1}^{n}\left(1+z_{1}^{2}\right)^{-1} z_{i},-2 \sigma^{-1} \sum_{i=1}^{n}\left(1+z_{1}^{2}\right)^{-1} z_{i} x_{i},-2 \sigma^{-1} \sum_{i=1}^{n}\left(1+z_{1}^{2}\right)^{-1} z_{i} \hat{z}_{i}\right],
\end{aligned}
$$

with elements denoted by

$$
\begin{aligned}
& \varphi_{1}(\theta)=-2 \sigma^{-1} \sum_{i=1}^{n}\left(1+z_{1}^{2}\right)^{-1} z_{i} \\
& \varphi_{2}(\theta)=-2 \sigma^{-1} \sum_{i=1}^{n}\left(1+z_{1}^{2}\right)^{-1} z_{i} x_{i} \\
& \varphi_{3}(\theta)=-2 \sigma^{-1} \sum_{i=1}^{n}\left(1+z_{1}^{2}\right)^{-1} z_{i} \hat{z}_{i} .
\end{aligned}
$$

Using these elements, the matrix $\varphi_{\theta}(\theta)$ is computed

$$
\varphi_{\theta}(\theta)=\left[\begin{array}{ccc}
\varphi_{1 \alpha}(\theta) & \varphi_{1 \beta}(\theta) & \varphi_{1 \sigma}(\theta) \\
\varphi_{2 \alpha}(\theta) & \varphi_{2 \beta}(\theta) & \varphi_{2 \sigma}(\theta) \\
\varphi_{3 \alpha}(\theta) & \varphi_{3 \beta}(\theta) & \varphi_{3 \sigma}(\theta)
\end{array}\right],
$$


where

$$
\begin{aligned}
& \varphi_{1 \alpha}(\theta)=\frac{\partial \varphi_{1}(\theta)}{\partial \alpha}=-2 \sigma^{-2} \sum_{i=1}^{n}\left[2\left(1+z_{i}^{2}\right)^{-2} z_{i}^{2}-\left(1+z_{i}^{2}\right)^{-1}\right] \\
& \varphi_{1 \beta}(\theta)=\frac{\partial \varphi_{1}(\theta)}{\partial \beta}=-2 \sigma^{-2} \sum_{i=1}^{n}\left[2\left(1+z_{i}^{2}\right)^{-2} z_{i}^{2} x_{i}-\left(1+z_{i}^{2}\right)^{-1} x_{i}\right] \\
& \varphi_{1 \sigma}(\theta)=\frac{\partial \varphi_{1}(\theta)}{\partial \sigma}=-4 \sigma^{-2} \sum_{i=1}^{n}\left[\left(1+z_{i}^{2}\right)^{-2} z_{i}^{3}-\left(1+z_{i}^{2}\right)^{-1} z_{i}\right] \\
& \varphi_{2 \alpha}(\theta)=\frac{\partial \varphi_{2}(\theta)}{\partial \alpha}=-2 \sigma^{-2} \sum_{i=1}^{n}\left[\varphi_{1 \alpha}(\theta) 2\left(1+z_{i}^{2}\right)^{-2} z_{i}^{2} x_{i}-\left(1+z_{i}^{2}\right)^{-1} x_{i}\right] \\
& \varphi_{2 \beta}(\theta)=\frac{\partial \varphi_{2}(\theta)}{\partial \beta}=-2 \sigma^{-2} \sum_{i=1}^{n}\left[2\left(1+z_{i}^{2}\right)^{-2} z_{i}^{2} x_{i}^{2}-\left(1+z_{i}^{2}\right)^{-1} x_{i}^{2}\right] \\
& \varphi_{2 \sigma}(\theta)=\frac{\partial \varphi_{2}(\theta)}{\partial \sigma}=-4 \sigma^{-2} \sum_{i=1}^{n}\left[2\left(1+z_{i}^{2}\right)^{-2} z_{i}^{3} x_{i}-\left(1+z_{i}^{2}\right)^{-1} z_{i} x_{i}\right] \\
& \varphi_{3 \alpha}(\theta)=\frac{\partial \varphi_{3}(\theta)}{\partial \alpha}=-2 \sigma^{-2} \sum_{i=1}^{n}\left[2\left(1+z_{i}^{2}\right)^{-2} z_{i}^{2} \hat{z}_{i}-\left(1+z_{i}^{2}\right)^{-1} \hat{z}_{i}\right] \\
& \varphi_{3 \beta}(\theta)=\frac{\partial \varphi_{3}(\theta)}{\partial \beta}=-2 \sigma^{-2} \sum_{i=1}^{n}\left[2\left(1+z_{i}^{2}\right)^{-2} z_{i}^{2} x_{i} \hat{z}_{i}-\left(1+z_{i}^{2}\right)^{-1} x_{i} \hat{z}_{i}\right] \\
& \varphi_{3 \sigma}(\theta)=\frac{\partial \varphi_{3}(\theta)}{\partial \sigma}=-4 \sigma^{-2} \sum_{i=1}^{n}\left[\left(1+z_{i}^{2}\right)^{-2} z_{i}^{3} \hat{z}_{i}-\left(1+z_{i}^{2}\right)^{-1} z_{i} \hat{z}_{i}\right] .
\end{aligned}
$$

The stand-in scalar canonical parameter can then be obtained as

$$
\chi(\theta)=\frac{\psi_{\varphi^{\prime}}\left(\hat{\theta}_{\psi}\right)}{\left\|\psi_{\varphi^{\prime}}\left(\hat{\theta}_{\psi}\right)\right\|} \varphi(\theta),
$$

where

$$
\psi_{\varphi^{\prime}}(\theta)=\psi_{\theta^{\prime}}(\theta) \varphi_{\theta^{\prime}}^{-1}(\theta)
$$

and

$$
\begin{gathered}
\psi_{\theta}(\theta)=\left(\begin{array}{lll}
0 & 1 & 0
\end{array}\right) . \\
Q=\operatorname{sgn}(\hat{\psi}-\psi)\left|\chi(\hat{\theta})-\chi\left(\hat{\theta}_{\psi}\right)\right|\left\{\frac{\left|\hat{\jmath}_{\varphi \varphi^{\prime}}(\hat{\theta})\right|}{\left|\hat{\jmath}_{\left(\lambda \lambda^{\prime}\right)}\left(\hat{\theta}_{\psi}\right)\right|}\right\}^{1 / 2} .
\end{gathered}
$$

To consider how different these higher order p-values can be from the standard p-values, we consider two examples for the Cauchy distribution. We make computer code available for Example 1 below. ${ }^{1}$

\footnotetext{
${ }^{1}$ Computer code using R Code is available from www.sfu.ca/ mrekkas for Example 1 as well as for two other examples (for the t-distribution and the Pareto distribution) that have not been included in the paper.
} 


\subsection{Example 1}

Consider the simple linear regression model

$$
y_{t}=\alpha+\beta x_{t}+\sigma \epsilon_{t}, \quad t=1,2, \ldots 7
$$

where $\epsilon$ is distributed as Cauchy. Suppose we have the following data:

\begin{tabular}{cccccccc}
$x$ & -3 & -2 & -1 & 0 & 1 & 2 & 3 \\
\hline$y$ & -4.28 & 0.89 & -10.42 & 1.30 & 4.56 & 1.73 & 8.49
\end{tabular}

The maximum likelihood estimate for the slope parameter is given as $\hat{\beta}=2.1065$. Table 1 below records the $\mathrm{p}$-values for testing different values of $\beta$ using the various methods. The two first-order methods represent the typical maximum likelihood departure reported in statistical packages which is denoted as MLE and the log-likelihood ratio in (2) which is denoted as LR. The two third-order p-values are obtained by using the Lugannani and Rice expression in (12) and the Barndorff-Nielsen expression given in (13).

Table 1: P-values for the Cauchy Distribution

\begin{tabular}{cccccc}
\hline Method & $\beta=0$ & $\beta=1.0$ & $\beta=1.5$ & $\beta=2.0$ & $\beta=2.5$ \\
\hline MLE & 0.0000 & 0.0000 & 0.0000 & 0.2340 & 0.0037 \\
LR & 0.0026 & 0.0239 & 0.0629 & 0.2933 & 0.0373 \\
Lugannani and Rice & 0.0093 & 0.0703 & 0.1227 & 0.2597 & 0.0359 \\
Barndorff-Nielsen & 0.0091 & 0.0681 & 0.1212 & 0.2598 & 0.0359 \\
\hline
\end{tabular}

Notes: P-values for various values of $\beta$ are reported using the maximum likelihood departure (MLE), the likelihood ratio (LR), the Lugannani and Rice expression and the Barndorff-Nielsen expression.

If we are interested in testing a null of $\beta=1$ or $\beta=1.5$, two different conclusions may be reached depending on which method was being used for inference. If the level of significance was chosen to be $5 \%$, one would reject the null of $\beta=1$ using either of the first-order methods while the null would not be rejected using either of the third-order methods. At this same level of significance, one would reject the null of $\beta=1.5$ using the MLE method, while failing to reject using any other of the methods.

\subsection{Example 2}

Suppose we consider the well known capital asset pricing model (CAPM) used in finance. This model relates a security's excess returns (relative to the risk-free rate) to the excess returns of the market. The econometric specification of this model is given as

$$
r_{j t}-r_{f t}=\alpha_{j}+\beta_{j}\left(r_{m t}-r_{f t}\right)+\epsilon_{j t},
$$

where $r_{j t}$ is the return on security $j$ at time $t, r_{f t}$ is the risk-free rate $f$ at time $t$, and $r_{m t}$ is the return on the market $m$ at time $t$. This model is outlined in Hill et al. (2001). Using monthly data over a 10-year period provided in Berndt (1991) (and used in Hill et al. (2001)) for monthly returns of Mobil Oil, we 
Figure 1: Approximations to the p-value function for the CAPM example

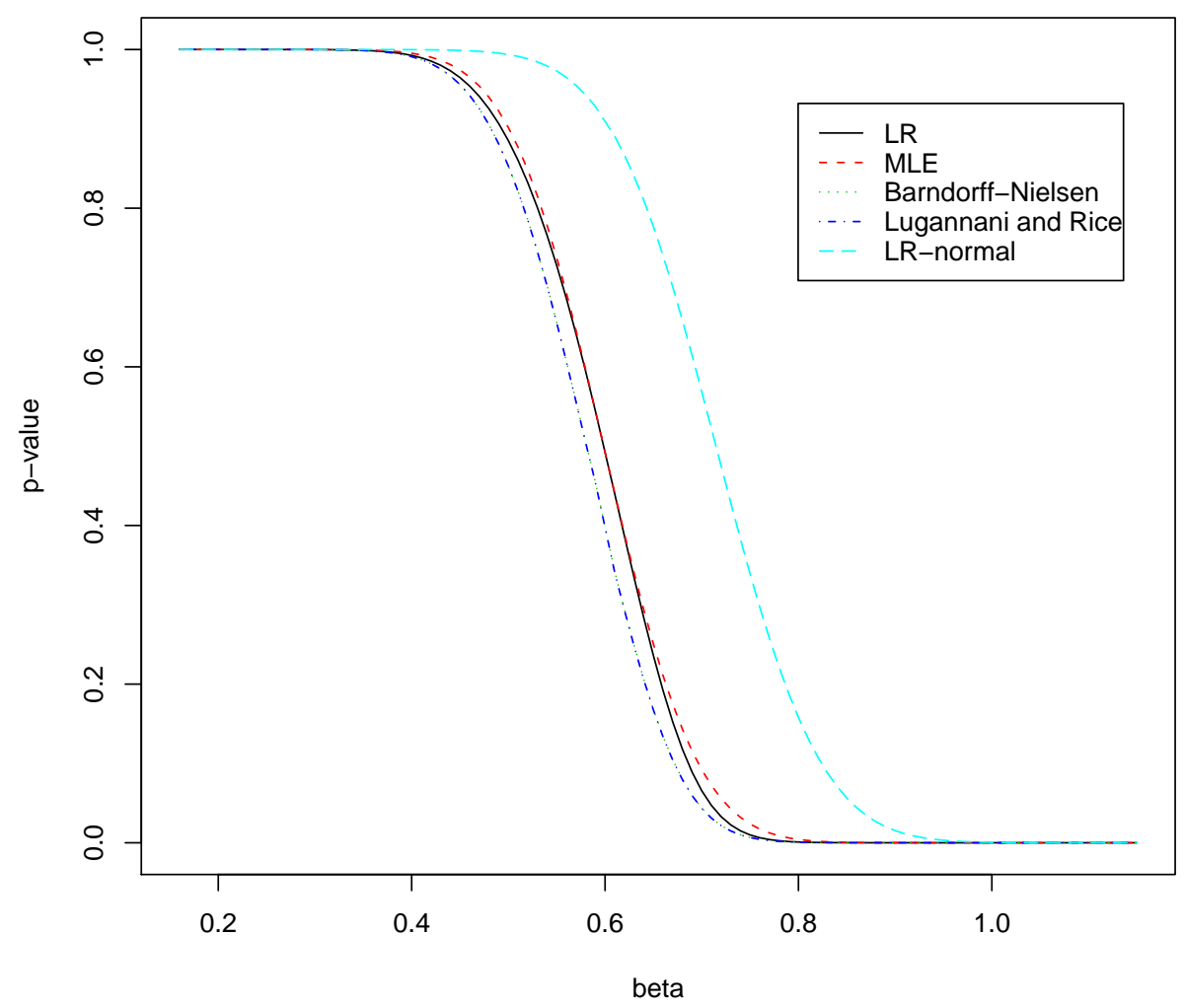

estimate the model above under the assumption of Cauchy errors. The maximum likelihood estimate $\hat{\alpha}$ is obtained as 0.0017 and the maximum likelihood estimate $\hat{\beta}$ is obtained as 0.5986 . Under the normal error assumption, the maximum likelihood estimates for $\alpha$ and $\beta$ are 0.0042 and 0.7147 , respectively. Testing a null hypothesis of $\alpha=0$ would yield the following p-values for each of the four methods: MLE $=0.6265, \mathrm{LR}=$ 0.6262, Lugannani and Rice $=0.6466$, and Barndorff-Nielsen $=0.6466$. Under the normal error assumption, the likelihood ratio method would produce a p-value of 0.7662 . For inference on the slope parameter, we plot the p-value functions resulting from each method in Figure 1. We also include the p-value approximation resulting from using the likelihood ratio method under the assumption of normal errors (LR - normal). It is clear from this figure that the methods produce different results, while the p-value functions for the Lugannani and Rice and Barndorff-Nielsen methods are indistinguishable in the figure, they are different from those functions produced by the LR and MLE first-order methods and very different from that produced by LR method assuming normal errors. Taking a particular example, if we are interested in testing a null hypothesis of $\beta=0.7$ the methods produce the following p-values: MLE $=0.0921, \mathrm{LR}=0.0656$, Lugannani and Rice $=0.0434$, and Barndorff-Nielsen $=0.0436$, and LR - normal $=0.5687$. For a $5 \%$ level of significance, the MLE, LR and LR - normal would not reject the null while the Lugannani and Rice and Barndorff-Nielsen methods would reject the null. 


\section{The Pareto Distribution}

The application of the third-order method to the Pareto distribution is novel to the literature. We consider the Pareto distribution for $y$,

$$
f(y)=\frac{\gamma^{\gamma+1} \exp (\beta x)}{[\gamma+y \exp (\beta x)]^{\gamma+1}}, \quad \quad y>0
$$

with

$$
F(y)=1-\frac{\gamma^{\gamma}}{[\gamma+y \exp (\beta x)]^{\gamma}}
$$

The $\log$-likelihood for $y_{1}, y_{2}, \ldots, y_{n}$ is given as

$$
l(\beta, \gamma)=\sum_{i=1}^{n}\left\{(\gamma+1) \log \gamma+\beta x_{i}-(\gamma+1) \log \left[\gamma+y_{i} \exp \left(\beta x_{i}\right)\right]\right\} .
$$

Setting the following first derivatives equal to zero and solving the system simultaneously yields the maximum likelihood estimate:

$$
\begin{aligned}
& l_{\beta}=\sum_{i=1}^{n} x_{i}-(\gamma+1) \sum_{i=1}^{n} \frac{y_{i} \exp \left(\beta x_{i}\right) x_{i}}{\left[\gamma+y_{i} \exp \left(\beta x_{i}\right)\right]} \\
& l_{\gamma}=n \log \gamma+n+\frac{n}{\gamma}-\sum_{i=1}^{n} \log \left[\gamma+y_{i} \exp \left(\beta x_{i}\right)\right]-(\gamma+1) \sum_{i=1}^{n} \frac{1}{\left[\gamma+y_{i} \exp \left(\beta x_{i}\right)\right]} .
\end{aligned}
$$

The second derivatives are obtained as follows:

$$
\begin{aligned}
& l_{\beta \beta}=-(\gamma+1) \gamma \sum_{i=1}^{n} \frac{y_{i} \exp \left(\beta x_{i}\right) x_{i}^{2}}{\left[\gamma+y_{i} \exp \left(\beta x_{i}\right)\right]^{2}} \\
& l_{\gamma \beta}=-\sum_{i=1}^{n} \frac{y_{i} \exp \left(\beta x_{i}\right) x_{i}}{\left[\gamma+y_{i} \exp \left(\beta x_{i}\right)\right]}+(\gamma+1) \sum_{i=1}^{n} \frac{y_{i} \exp \left(\beta x_{i}\right) x_{i}}{\left[\gamma+y_{i} \exp \left(\beta x_{i}\right)\right]^{2}} \\
& l_{\gamma \gamma}=\frac{n}{\gamma}-\frac{n}{\gamma^{2}}-\sum_{i=1}^{n} \frac{2}{\left[\gamma+y_{i} \exp \left(\beta x_{i}\right)\right]}+(\gamma+1) \sum_{i=1}^{2} \frac{1}{\left[\gamma+y_{i} \exp \left(\beta x_{i}\right)\right]^{2}} .
\end{aligned}
$$

For the canonical parameterization, the quantity $V$ is required,

$$
\begin{aligned}
V & =\left.\frac{\partial y}{\partial \theta^{\prime}}\right|_{\hat{\theta}}=\left.\left(\frac{\partial y}{\partial \beta}, \frac{\partial y}{\partial \gamma}\right)\right|_{\hat{\theta}} \\
& =\left.\left(-y x, \quad \frac{\{[(1+\log \gamma)-\log [\gamma+y \exp (\beta x)]][\gamma+y \exp (\beta x)]-\gamma\}}{\gamma \exp (\beta x)}\right)\right|_{\hat{\theta}} \\
& =\left(-y x, \quad \frac{\{[(1+\log \hat{\gamma})-\log [\hat{\gamma}+y \exp (\hat{\beta} x)]][\hat{\gamma}+y \exp (\hat{\beta} x)]-\hat{\gamma}\}}{\hat{\gamma} \exp (\hat{\beta} x)}\right) \\
& =\frac{\partial l}{\partial y_{i}}=-(\gamma+1) \frac{\exp \left(\beta x_{i}\right)}{\left[\gamma+y_{i} \exp \left(\beta x_{i}\right)\right]}
\end{aligned}
$$


The reparameterization is given by

$$
\varphi^{\prime}(\theta)=\left[\frac{\partial l}{\partial y}\right]\left[-y x, \quad \frac{\left\{\left[(1+\log \hat{\gamma})-\log \left[\hat{\gamma}+y_{i} \exp \left(\hat{\beta} x_{i}\right)\right]\right]\left[\hat{\gamma}+y_{i} \exp \left(\hat{\beta} x_{i}\right)\right]-\hat{\gamma}\right\}}{\hat{\gamma} \exp \left(\hat{\beta} x_{i}\right)}\right],
$$

with elements denoted by

$$
\begin{aligned}
& \varphi_{1}(\theta)=(\gamma+1) \sum_{1=1}^{n} \frac{\exp \left(\beta x_{i}\right) y_{i} x_{i}}{\left[\gamma+y_{i} \exp \left(\beta x_{i}\right)\right]} \\
& \varphi_{2}(\theta)=-(\gamma+1) \sum_{1=1}^{n} \frac{\exp \left(\beta x_{i}\right)}{\left[\gamma+y_{i} \exp \left(\beta x_{i}\right)\right]} \frac{\left\{\left[(1+\log \hat{\gamma})-\log \left[\hat{\gamma}+y_{i} \exp \left(\hat{\beta} x_{i}\right)\right]\right]\left[\hat{\gamma}+y_{i} \exp \left(\hat{\beta} x_{i}\right)\right]-\hat{\gamma}\right\}}{\hat{\gamma} \exp \left(\hat{\beta} x_{i}\right)} .
\end{aligned}
$$

Using these elements, the matrix $\varphi_{\theta}(\theta)$ can be computed

$$
\varphi_{\theta}(\theta)=\left[\begin{array}{cc}
\varphi_{1 \beta}(\theta) & \varphi_{1 \gamma}(\theta) \\
\varphi_{2 \beta}(\theta) & \varphi_{2 \gamma}(\theta)
\end{array}\right]
$$

where

$$
\begin{aligned}
\frac{\partial \varphi_{1}(\theta)}{\partial \beta}= & (\gamma+1) \gamma \sum_{i=1}^{n} \frac{\exp \left(\beta x_{i}\right) y_{i} x_{i}^{2}}{\left[\gamma+y_{i} \exp \left(\beta x_{i}\right)\right]^{2}} \\
\frac{\partial \varphi_{1}(\theta)}{\partial \gamma}= & \sum_{i=1}^{n} \frac{\exp \left(\beta x_{i}\right) y_{i} x_{i}}{\left[\gamma+y_{i} \exp \left(\beta x_{i}\right)\right]}-(\gamma+1) \sum_{i=1}^{n} \frac{\exp \left(\beta x_{i}\right) y_{i} x_{i}}{\left[\gamma+y_{i} \exp \left(\beta x_{i}\right)\right]^{2}} \\
\frac{\partial \varphi_{2}(\theta)}{\partial \beta}= & -(\gamma+1) \gamma \sum_{i=1}^{n} \frac{\exp \left(\beta x_{i}\right) x_{i}}{\left[\gamma+y_{i} \exp \left(\beta x_{i}\right)\right]^{2}} \times \\
& \frac{\left\{\left[(1+\log \hat{\gamma})-\log \left[\hat{\gamma}+y_{i} \exp \left(\hat{\beta} x_{i}\right)\right]\right]\left[\hat{\gamma}+y_{i} \exp \left(\hat{\beta} x_{i}\right)\right]-\hat{\gamma}\right\}}{\hat{\gamma} \exp \left(\hat{\beta} x_{i}\right)} \\
\frac{\partial \varphi_{2}(\theta)}{\partial \gamma}= & -\sum_{i=1}^{2} \frac{\exp \left(\beta x_{i}\right)\left[y_{i} \exp \left(\beta x_{i}\right)-1\right]}{\left[\gamma+y_{i} \exp \left(\beta x_{i}\right)\right]^{2}} \times \\
& \frac{\left\{\left[(1+\log \hat{\gamma})-\log \left[\hat{\gamma}+y_{i} \exp \left(\hat{\beta} x_{i}\right)\right]\right]\left[\hat{\gamma}+y_{i} \exp \left(\hat{\beta} x_{i}\right)\right]-\hat{\gamma}\right\}}{\hat{\gamma} \exp \left(\hat{\beta} x_{i}\right)} .
\end{aligned}
$$

The canonical parameter is then given by

$$
\chi(\theta)=\frac{\psi_{\varphi^{\prime}}\left(\hat{\theta}_{\psi}\right)}{\left\|\psi_{\varphi^{\prime}}\left(\hat{\theta}_{\psi}\right)\right\|} \varphi(\theta),
$$

where

$$
\psi_{\varphi^{\prime}}(\theta)=\psi_{\theta^{\prime}}(\theta) \varphi_{\theta^{\prime}}^{-1}(\theta)
$$

and

$$
\psi_{\theta}(\theta)=\left(\begin{array}{ll}
1 & 0
\end{array}\right)
$$




$$
Q=\operatorname{sgn}(\hat{\psi}-\psi)\left|\chi(\hat{\theta})-\chi\left(\hat{\theta}_{\psi}\right)\right|\left\{\frac{\left|\hat{\jmath}_{\varphi \varphi^{\prime}}(\hat{\theta})\right|}{\left|\hat{\jmath}_{\left(\lambda \lambda^{\prime}\right)}\left(\hat{\theta}_{\psi}\right)\right|}\right\}^{1 / 2}
$$

\section{Conclusion}

The higher order likelihood-based inference approach developed by Fraser and Reid (1995) was applied to three heavy-tailed distributions. A systematic exposition of the necessary calculations was provided for the interested applied researcher as a guide to implement this procedure. 


\section{References}

[1] Barndorff-Nielsen, O., 1986, Inference on Full or Partial Parameters Based on the Standardized Signed Log Likelihood Ratio, Biometrika 73, 307-22.

[2] Barndorff-Nielsen, O., 1991. Modified Signed Log-Likelihood Ratio, Biometrika 78, 557-563.

[3] Bedard, M., Fraser, D., Wong, A., 2007. Higher Accuracy for Bayesian and Frequentist Inference: Large Sample Theory for Small Sample Likelihood, Statistical Science, forthcoming.

[4] Berndt, E., 1991. The Practice of Econometrics: Classic and Contemporary, Addison-Wesley, New York.

[5] Brazzale, A., 1999. Approximate conditional inference in logistic and loglinear models, Journal of Computational and Graphical Statistics 8, 653-661.

[6] Brazzale, A., Davison, A., Reid, N., 2007. Applied Asymptotics: Case Studies in Small-Sample Statistics, Cambridge Series in Statistical and Probabilistic Mathematics.

[7] Fraser, D., Reid, N., 1995. Ancillaries and Third Order Significance, Utilitas Mathematica 47, 33-53.

[8] Fraser, D., Reid, N., Wu, J., 1999. A Simple General Formula for Tail Probabilities for Frequentist and Bayesian Inference, Biometrika 86, 249-264.

[9] Fraser, D., Wong, A., Wu, J., 1999. Regression Analysis, Nonlinear or Nonnormal: Simple and Accurate p Values From Likelihood Analysis, Journal of the American Statistical Association 94(448), 1286-1295.

[10] Hill, R., Griffiths, W., Judge, G., 2001 Undergraduate Econometrics, Wiley, New York.

[11] Lugannani, R., Rice, S., 1980. Saddlepoint Approximation for the Distribution of the Sums of Independent Random Variables, Advances in Applied Probability 12, 475-490.

[12] Taback, N., 2002. Small Sample Inference in Location Scale Models with Student $(\lambda)$ Errors, Communications in Statistics: Simulation and Computation 31, 557-566. 